\title{
Lessons Learned from using a Cs-Corrected, Energy-Filtered, Phase-Plate TEM for Single-Particle CryoEM
}

\author{
Anchi Cheng ${ }^{1,2}$, Edward Eng ${ }^{1}$, William Rice ${ }^{1}$, Laura Kim ${ }^{1}$, Michael Alink ${ }^{3}$, Clinton S. Potter ${ }^{1,2}$ and \\ Bridget Carragher ${ }^{1,2}$. \\ 1. Simons Electron Microscopy Center, New York Structural Biology Center, New York, New York \\ 2. National Resource for Automated Molecular Microscopy, New York, New York \\ 3. Thermo Fisher Scientific, Materials \& Structural Analysis, Hillsboro, Oregon
}

Single particle cryo-electron microscopy (SP cryoEM) of frozen-hydrated biological complexes has benefited from several new technological developments. Recent advances in direct electron detectors, stable thermal and electron optics, as well as improved software algorithms and the availability of powerful computational hardware means that reconstructions to near-atomic resolution can now be routinely achieved for suitable samples.

Phase plates (PP) provide for high contrast at low resolutions crucial for alignment of small particles without the need to apply high levels of defocus. At an optimal $90^{\circ}$ phase shift, the contrast at close to zero spatial frequency is maximized. Even with only a $20^{\circ}$ phase shift, a $\sim 5$ fold contrast enhancement is achieved for vitrified particles. The recently available Volta PP [1] is a practical hole-free phase plate which however induces a continuous phase shift while it is exposed to the focused electron beam. Workflows have been successfully established to use the Volta PP to acquire high-resolution images for SP cryoEM using either small defocus [2] or in-focus [1] imaging conditions.

Energy filters remove inelastic-scattered electrons before they reach the detector. Energy filters are usually not considered critical for SP cryoEM because the ice the protein particles are embedded in is usually relatively thin and thus does not contributed a major percentage of inelastically scattered electrons. Nevertheless, the images obtained using the energy filter have better contrast, and thus theoretically may also help to improve particle alignments and thus ultimately map resolution [3].

The spherical aberration (Cs) of the objective lens can be minimized with an image corrector which can also minimize several other common aberrations. The Cs corrector can also be trained to minimize these aberrations in response to optical changes such as an applied image shift. The Cs corrector has the highest impact on the obtainable resolution for in-focus images and thus matched well with the need for in-focus PP imaging (Figure 1).

We recently acquired an FEI Titan Krios equipped with an image Cs corrector, energy filter, and Volta phase plate. Using the $20 \mathrm{~S}$ proteasome, glutamate dehydrogenase, and other protein complexes, we have explored the value of a variety of imaging parameters and instrument conditions including energy filter slit width, defocus, Cs value and other parameters adjustable using the Cs corrector in combination with the PP. We have also developed a workflow and algorithm to evaluate and increase productivity of these devices when collecting data using Leginon [4]. We have learned that each type of specimen requires a different imaging strategy. For example, small defocus PP data collection is efficient when the protein concentration allows for accurate CTF estimation, including the phase shift. However, with low concentrations of protein, it is preferable to acquire images in-focus with the PP in order to 
maximize the number of usable images at high resolution. The energy filter with its slit aligned for zero-loss peak increases the resolution obtained for the $20 \mathrm{~S}$ proteasomes.

In this presentation we will discuss these and other lessons we have learned from these systematic studies of test samples image under a variety of conditions [5].

[1] R. Danev and W. Baumeister elife 5 (2016), e1306.

[2] R. Danev et al, elife 6 (2017) e23006.

[3] K. Yonekura et al, Journal of Structural Biology 156 (2006), p. 524.

[4] C. Sulloway et al, Journal of Structural Biology 159 (2007), p. 335.

[5] The authors acknowledge funding from NIH National Institute of General Medical Sciences

(GM103310) and the Simons Foundation (349247).

Figure 1. In-focus phase plate image of frozen-hydrated 20S proteasome taken on Cs corrected, energyfiltered (15 eV slit-width) TEM. The power spectrum of this image shown on the right displays no Thon rings, consistent with the in-focus imaging condition. Sample generously provided by Yifan Cheng's group.
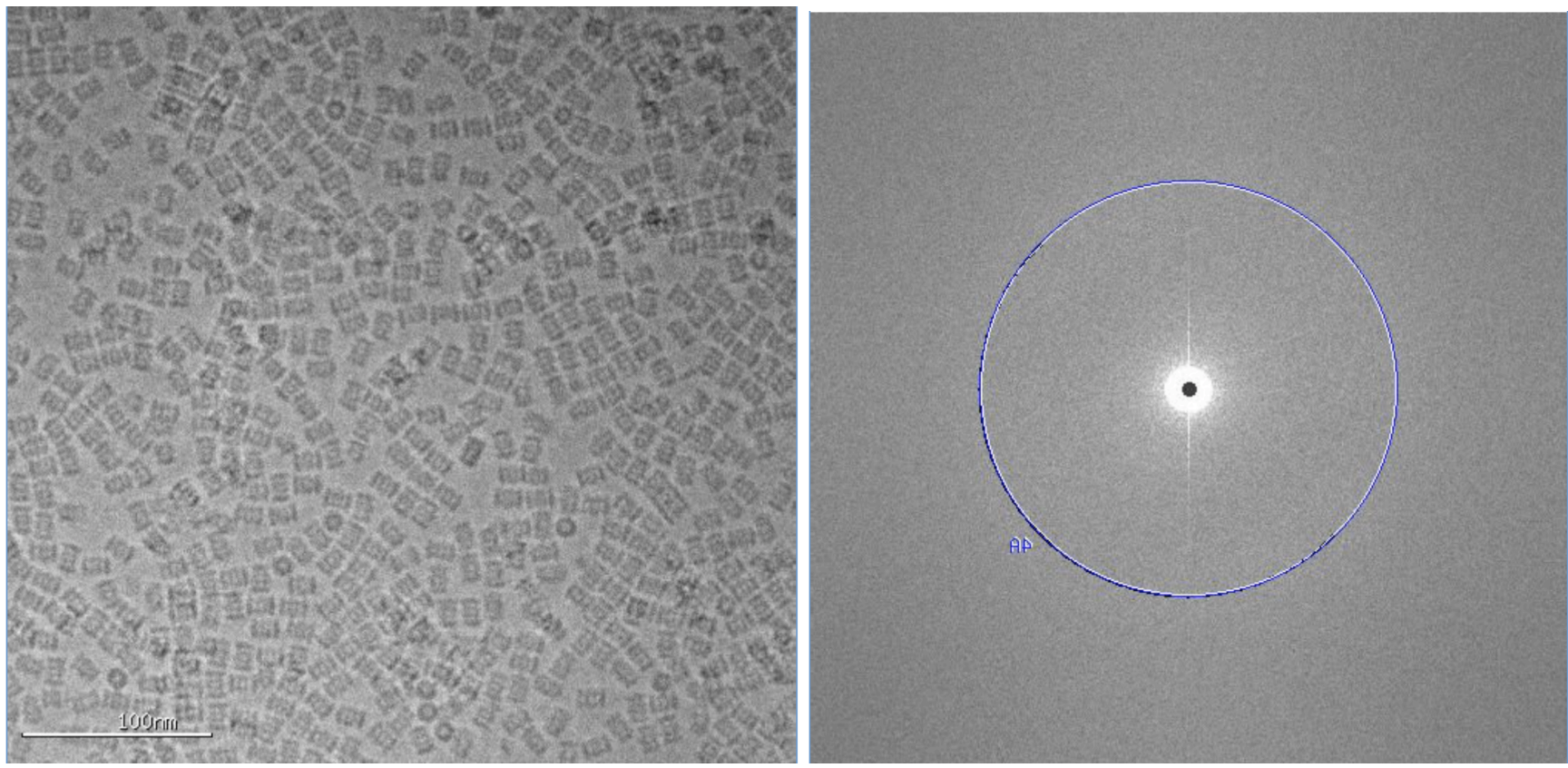